\title{
Impact of ERAS compliance on the delay between surgery and adjuvant chemotherapy in hepatobiliary and pancreatic malignancies
}

\author{
Pénélope St-Amour ${ }^{1}$ • Pascal St-Amour ${ }^{2} \cdot$ Gaëtan-Romain Joliat $^{1} \cdot$ Aude Eckert $^{3} \cdot$ Ismail Labgaa $^{1} \cdot$ Didier Roulin $^{1}$. \\ Nicolas Demartines ${ }^{1} \cdot$ Emmanuel Melloul $^{1}$
}

Received: 23 April 2020 / Accepted: 1 September 2020 / Published online: 11 September 2020

(C) The Author(s) 2020

\begin{abstract}
Background Multidisciplinary approach with adjuvant chemotherapy is the key element to provide optimal outcomes in pancreas and liver malignancies. However, post-operative complications may increase the interval between surgery and chemotherapy with negative oncologic effects.

Hypothesis and study aim The aim of the study was to analyse whether compliance to Enhanced Recovery After Surgery (ERAS) pathway was associated with decreased interval to adjuvant chemotherapy.

Methods Retrospective analysis of all consecutive ERAS patients with surgery for hepatobiliary or pancreatic malignancies at the University Hospital of Lausanne between 2012 and 2016. Multivariate analysis was performed to assess the impact of ERAS compliance on time to chemotherapy.

Results A total of 133 patients with adjuvant chemotherapy were included ( $n=44$ liver and $n=89$ pancreatic cancer). Median compliance to ERAS was 61\% (IQR 55-67) for the study population, and median delay to chemotherapy was 49 days (IQR 3961). Overall, compliance $\geq 67 \%$ to ERAS induced a significant reduction in the interval between surgery and chemotherapy for young patients ( $<65$ years old) with or without severe comorbidities (reduction of 22 and 10 days, respectively). High compliance in young ASA3 patients with liver colorectal metastases was associated with an increase of 481 days of DFS.

Conclusions ERAS compliance $\geq 67 \%$ tends to be associated with a reduction in the delay to adjuvant chemotherapy for young patients with hepatobiliary and pancreatic malignancies. More prospective studies with strict adhesion to the ERAS protocol are needed to confirm these results.
\end{abstract}

Keywords ERAS · Liver surgery $\cdot$ Pancreatic surgery $\cdot$ Adjuvant chemotherapy

\begin{tabular}{llll}
\multicolumn{2}{l}{ Abbreviation } & DFS & Disease-free survival \\
ASA & American Society of & EIAS & ERAS Interactive Audit System \\
& Anesthesiology & ERAS & Enhanced Recovery After Surgery \\
CCI & Comprehensive Complication & MDT & Multidisciplinary tumour board \\
& Index & OS & Overall survival
\end{tabular}

Nicolas Demartines

demartines@chuv.ch

1 Department of Visceral Surgery, Lausanne University Hospital (CHUV), Rue du Bugnon 46, 1011 Lausanne, Switzerland

2 Department of Economics, HEC Lausanne, University of Lausanne (UNIL), Lausanne, Switzerland

3 Department of Biology and Medicine, University of Lausanne (UNIL), Lausanne, Switzerland

\section{Introduction}

Pancreas and liver malignancies are common tumours with increasing incidence and poor prognosis. Five-year survival is $9 \%$ for all stages of pancreas malignancies and $18 \%$ for liver malignancies [1]. To improve this unfavourable outcome, multidisciplinary approach including neo- or adjuvant chemotherapy combined with surgical resection has become the best 
treatment to offer long-term disease-free (DFS) and overall survival (OS).

The ESPAC-3 trial showed that the most important factor for long-term survival in pancreas adenocarcinoma was to achieve complete cycles of adjuvant chemotherapy. However, if it is not possible to undergo full cycles, the recommended strategy is to wait for a full post-operative recovery rather than to start early without being able to finish the treatment [2]. On the other hand, delayed chemotherapy after 12 weeks would not be associated with a decreased long-term survival for pancreatic cancer, because adjuvant chemotherapy is a prognostic factor by itself, so it should be offered even later [3]. For colorectal cancer and its liver metastases, it is well demonstrated that adjuvant chemotherapy should be initiated within 6 to 8 post-operative weeks to offer the best longterm outcome [4-6]. Therefore, the timing for adjuvant chemotherapy is one of the key elements in patients with hepatobiliary and pancreatic malignancies. For this reason, recovery after surgery should be optimal to allow chemotherapy beginning as soon as the patients are fit enough.

Enhanced Recovery After Surgery (ERAS) pathway has been developed to reduce the negative effect of the surgical stress. Numerous meta-analyses have shown the benefits of an ERAS pathway implementation in pancreas and liver surgery [7-11]. The principal advantages are a reduction in medical complications after surgery, reduced length of stay and decreased costs. Guidelines for ERAS protocol are based on these studies and applied in many hospitals worldwide [12-14]. Achieving the same goals, recent studies have shown the benefits of introducing other similar pathways, especially targeting perioperative rehabilitation and more precisely prehabilitation [15].

The question remains whether ERAS pathways have an impact on the time to receipt of adjuvant chemotherapy. Moreover, data on the association between the compliance to ERAS and the increase of DFS and OS are scarce.

The aim of this study was to analyse the impact of ERAS compliance on the delay to adjuvant chemotherapy in patients operated for liver and pancreas malignancies.

\section{Material and methods}

\section{Data}

This is a retrospective analysis of a prospectively maintained database. All included patients underwent pancreatic or liver surgery for malignancies between 2012 and 2016, and all were included in an ERAS protocol $[12,13]$. In addition, they all were discussed in multidisciplinary tumour board (MDT) meeting and had an indication to receive adjuvant chemotherapy after surgery. Tumours included were pancreas ductal adenocarcinoma, cholangiocarcinoma, liver colorectal metastasis and gallbladder adenocarcinoma. Patients with liver colorectal metastases who benefitted from a reverse treatment without chemotherapy between the hepatic and colorectal surgery were excluded from the analysis $(n=15)$. Pre-operative patients' characteristics such as age, gender, BMI, smoking and alcohol drinking habits and ASA score (American Society of Anesthesiology) were included as cofactors. Post-operative data, such as CCI score (Comprehensive Complication Index), were also used as explanatory variables. Following standard practices, patients who died within 90 days after surgery were excluded of the survival analysis, since their death was more likely linked to the complications of surgery than to the oncological disease [16-18].

Adjuvant chemotherapy was defined as systemic oncologic treatment (chemotherapy) initiated within 200 days after surgery as previously defined [3, 19]. If chemotherapy was initiated after a recurrence of the disease, it was considered as a palliative treatment and thus not considered as adjuvant. These cases were excluded from the analysis $(n=10)$. Total compliance to ERAS pathway was calculated by a software program developed by ERAS society to provide a numerical score to compliance, taking into account pre-admission and pre-, intraand post-operative compliances (EIAS: ERAS Interactive Audit System). Measurement of these compliances is principally based on data concerning the patients' hospitalisation (i.e. post-operative early refeeding and mobilisation, weight's evolution), collected by nurses and medical assistance personnel. Data concerning patients' disease and perioperative information (i.e. nausea prevention, surgical duration) are also collected by the anaesthetist and the surgeon. The patient is himself strongly involved, during all the process. All this information is then summarised by an ERAS dedicated clinical nurse in a form on the software, and the percentage of compliances is generated. This study was approved by the local Ethics' Commission (Project-ID 2019-01097).

\section{Outcomes analysed}

The primary outcome analysed was the delay (measured in days) between the date of surgery and initiation of the first cycle of adjuvant chemotherapy.

\section{Statistical analyses}

Multivariate regression analysis (using STATA@ software) was used to study the effects of high compliance with ERAS guidelines on the time to receipt of adjuvant chemotherapy. More precisely, we resorted to a maximum likelihood estimation of a fully parameterised survival time (ST) model for the time to adjuvant chemotherapy, assuming a Weibull distribution. The uncensored sample was restricted to the observations with achieved outcome (e.g. 133 out of 173 cases received adjuvant chemotherapy). For robustness and comparison with 
the literature, this multivariate procedure was also applied for two other outcomes of interest, time to recurrence and time to death in order to analyse DFS and OS [2, 3, 20].

In order to control for patient-specific characteristics, clinically relevant covariates were included such as gender, as well as health-related habits (smoking, alcohol drinking, high BMI and CCI as well as ASA score), in the time to outcome analysis. To facilitate the interpretation of the analysis, binary variables relying on the 75th percentile for BMI, ERAS compliance and $\mathrm{CCI}$ were generated (describing the higher part of the distribution). The main regressor of interest was high compliance to ERAS and was thus considered as a compliance of $\geq 67 \%$ (75th percentile). It was interacted with an old age indicator (age $>65$ years old), as it is known that age is related to worse survival, especially after pancreatic cancer surgery $[21,22]$. The use of interaction terms allowed calculating the effects of high compliance for the entire data sample, as well as for sub-groups stratified by age. We also considered a triple interaction of compliance, age and ASA in additional testing, and all our main results were robust to this alternative specification.

In the attempt to compare our results with available literature, we also tested the same analyses with a threshold of a hundred days for initiation of adjuvant chemotherapy [2, 23], and the results were qualitatively similar to those mentioned here.

Moreover, in order to control for the type of illness heterogeneity, we performed two types of analyses. First, the type of malignancy (liver versus pancreas) was included as a regressor interacted with compliance to differentiate the effect of compliance by type of cancer. Our results were qualitatively similar. Second, we also analysed heterogeneity by separate regressions by type of cancer subgroups (liver colorectal metastases versus pancreas adenocarcinomas). The associated results are discussed below.

The reported regression output refers to the marginal effect of high compliance, measured in changes in median time to outcome.

\section{Results}

\section{Descriptive statistics}

Figure 1 describes the study flow chart. Among patients included in the ERAS database, 227 had surgery for pancreatic or liver malignancies defined by the inclusion criteria between 2012 and $2016(n$ pancreas $=127 ; n$ liver $=100)$. Among them, 173 had an indication to adjuvant chemotherapy after MDT discussion. Finally, 40 patients (23\%) did not receive the adjuvant treatment within 200 days after surgery because of comorbidities $(n=7)$, progression of the disease after post-operative imaging reassessment $(n=15)$, complications $(n=8)$ or early death $(n=2)$ (unknown reason in 8 cases). These patients had a median compliance to ERAS of 59\% (IQR 52-64\%). The main study group therefore included 133 patients who received adjuvant chemotherapy, at a known date $(n$ pancreas $=89 ; n$ liver $=44)$. In the pancreas group, all the patients had adenocarcinoma. In the liver group, 37 patients had colorectal metastases, one patient had intrahepatic cholangiocarcinoma, five patients had hilar cholangiocarcinoma and one patient had gallbladder adenocarcinoma. Concerning the liver colorectal metastases, the majority $(n=30$, $81 \%)$ had synchronous lesions. Only one fourth $(n=9,24 \%)$ benefitted from a reverse treatment, and only 2 patients (5\%) had a one-stage procedure. Median compliance to ERAS for patients with liver malignancies was $100 \%$ pre-admission (IQR 58-100\%), 86\% pre-operative (IQR 86-88\%), 67\% intra-operative (IQR $57-71 \%$ ) and $28 \%$ post-operative (IQR $15-46 \%)$. For pancreas malignancies, it was $100 \%$ preadmission (IQR 67-100\%), 100\% pre-operative (IQR 100$100 \%$ ), $100 \%$ intra-operative (IQR $75-100 \%$ ) and $36 \%$ postoperative (IQR 20-46\%). Due to the low number of cases in each group, more analysis could not be performed. Descriptive characteristics of the patients are summarised in Table 1.

For all the study population who received adjuvant chemotherapy $(n=133)$, the median time between surgery and receipt

Table 1 Patients' characteristics

\begin{tabular}{llll}
\hline Variables & Liver $n=62(36 \%)$ & Pancreas $n=111(64 \%)$ & All $n=173(100 \%)$ \\
\hline Female & $23(37 \%)$ & $60(54 \%)$ & $83(48 \%)$ \\
Smoking & $23(37 \%)$ & $32(29 \%)$ & $55(32 \%)$ \\
Alcohol & $8(13 \%)$ & $12(11 \%)$ & $20(12 \%)$ \\
ASA 3 & $17(27 \%)$ & $43(39 \%)$ & $60(35 \%)$ \\
Age (years) & $63($ IQR 55-69) & $68($ IQR 61-73) & 67 (IQR 58-72) \\
BMI (kg/m $\left.{ }^{2}\right)$ & $25($ IQR 23-28) & $25($ IQR 23-28) & 25 (IQR 23-28) \\
ERAS compliance $(\%)$ & $54($ IQR 48-61) & 63 (IQR 58-70) & 61 (IQR 55-67) \\
CCI & $0($ IQR 0-30) & 23 (IQR 12-39) & 21 (IQR 0-35) \\
\hline
\end{tabular}

Patients' demographic characteristics for all the study population and by subgroup of liver or pancreas malignancies

ASA American Society of Anesthesiologists, BMI body mass index, ERAS Enhanced Recovery After Surgery, CCI Comprehensive Complication Index 
Fig. 1 Flowchart. Description of patients' inclusion in the study group for analysis. Adj adjuvant, Chemo chemotherapy, MDT multidisciplinary tumour board meeting

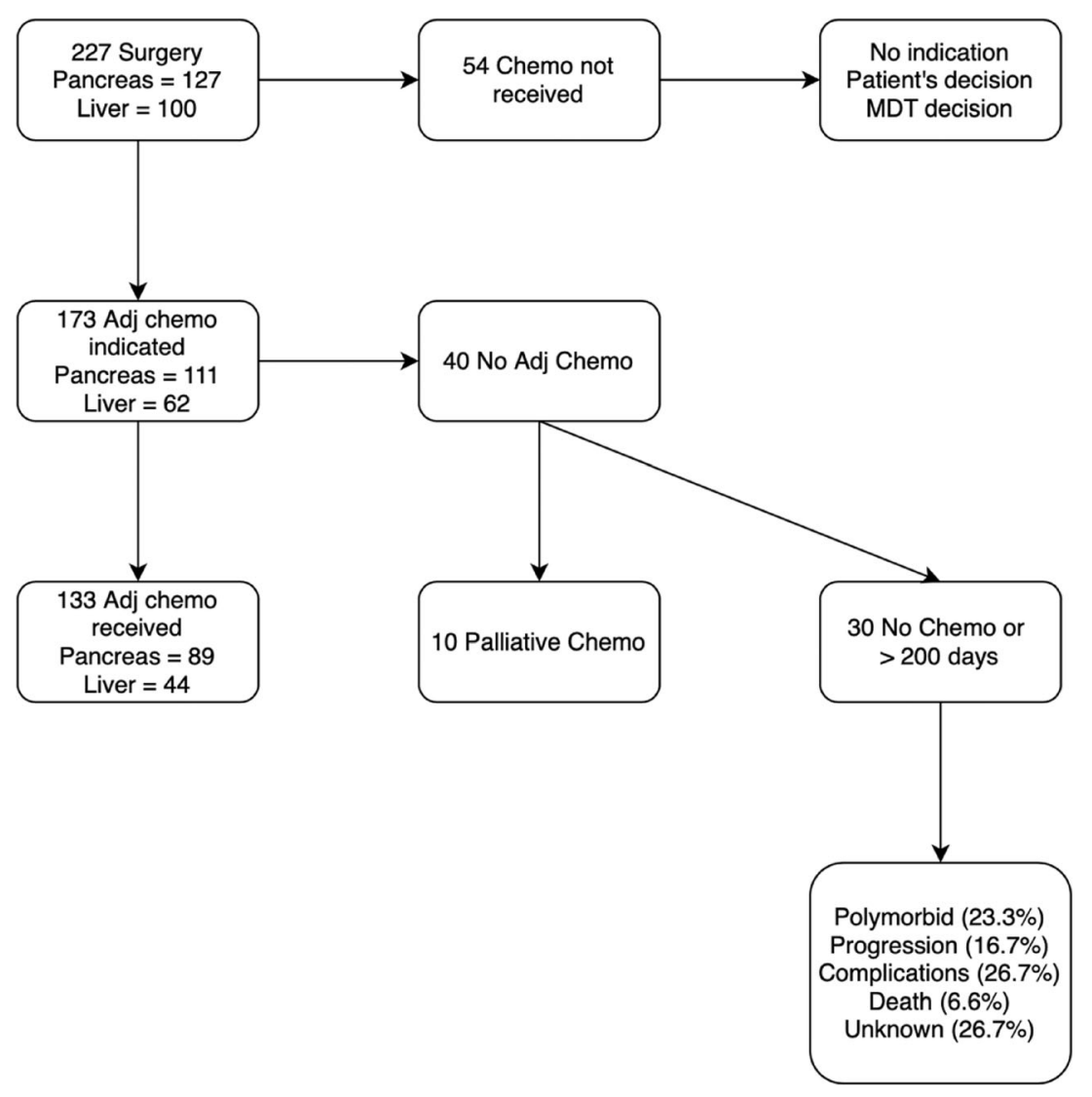

of adjuvant chemotherapy was 49 days (IQR 39-61). Median DFS was 13 months (IQR 5-22), and OS was 34 months (IQR 25-42) for the patients with liver colorectal metastases. Patients with pancreas malignancies had a median DFS of 12 months (IQR 8-22) and median OS of 20 months (IQR 13-36).

Compliance to ERAS was comparable $(p=0.25)$ in patients $<65$ years old (median compliance $61 \%$ ) and $>65$ years old (median 62\%). Moreover, it was also comparable regarding the ASA score (median compliance $61 \%$ for ASA1-2 and ASA $3, p=0.35$ ) and the CCI score (median compliance $58 \%$ for high CCI, $61 \%$ for low CCI, $p=0.10$ ).

\section{Multivariate analyses}

\section{Time to adjuvant chemotherapy}

\section{Overall population}

Table 2 displays the marginal effects (measured in changes in median waiting time) of the explanatory variables on the time to receipt of adjuvant chemotherapy for all the study population (i.e. exogenous variables not affected by our outcomes variables). Compliance $>67 \%$ to ERAS had no effect on the
Table 2 Time to chemotherapy (all patients)

\begin{tabular}{lllllll}
\hline Variables & Chg. med. time & Std. err. & $z$ & $P>z$ & \multicolumn{2}{l}{$95 \%$ conf. interval } \\
\hline Female & 6.209 & 2.983 & 2.080 & 0.037 & 0.361 & 12.056 \\
High BMI & -1.539 & 3.211 & -0.480 & 0.632 & -7.832 & 4.754 \\
Smoker & -6.346 & 3.028 & -2.100 & 0.036 & -12.280 & -0.412 \\
Alcohol drinking & 12.121 & 4.122 & 2.940 & 0.003 & 4.043 & 20.199 \\
High CCI & 7.820 & 3.554 & 2.200 & 0.028 & 0.854 & 14.786 \\
High compliance & -4.490 & 3.018 & -1.490 & 0.137 & -10.404 & 1.425 \\
Age $>$ 65 & 0.412 & 3.518 & 0.120 & 0.907 & -6.483 & 7.306 \\
ASA3 & 13.646 & 5.120 & 2.670 & 0.008 & 3.611 & 23.681 \\
\hline
\end{tabular}

Multivariate analysis of time to chemotherapy for all the study population

$B M I$ body mass index, CCI Comprehensive Complication Index, ASA American Society of Anesthesiologists, Chg. Med. Time change in median time, Std. Err standard error, 95\% Conf. Interval 95\% confidence interval 


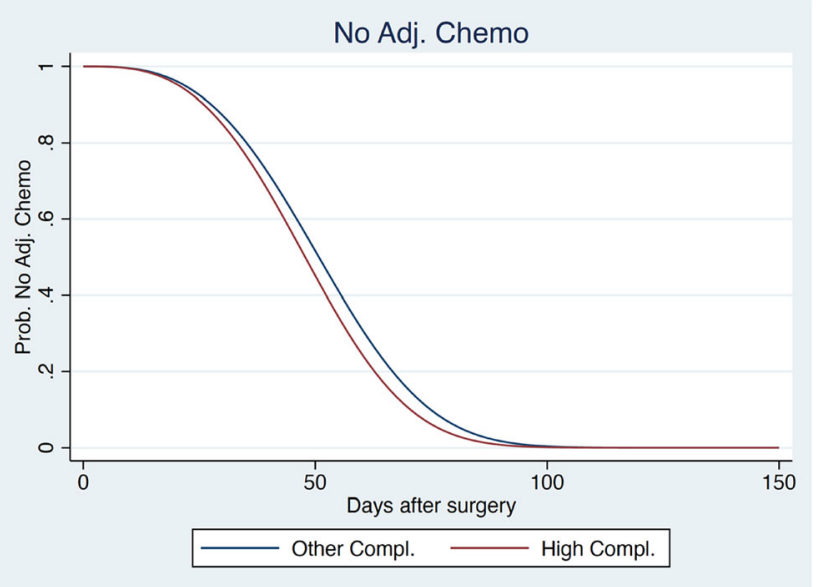

Fig. 2 Probability no adjuvant chemotherapy for all study population. Weibull distribution of the probability not to have an adjuvant chemotherapy at each moment after surgery in days, for high compliers (compliance $\geq 67 \%$ ) versus other compliances, for all patients with hepatobiliary and pancreas malignancies. Note that for this graph, $n$ Low Compl. $=91$ and $n$ High Comp. $=42, p=0.37$. Adj chemo adjuvant chemotherapy, Compl compliance, Prob probability

time to receipt of adjuvant chemotherapy when considering the whole study population (Fig. 2).

When stratified by age, the subgroup analysis showed that compliance $>67 \%$ to ERAS reduced the interval to chemotherapy of 12 days for younger patients ( $<65$ years old, $p=0.001$ ) (Table 3). The effect on shorter waiting time is illustrated in Fig. 3 , which plots the probability of no adjuvant chemotherapy, in function of days after surgery, with the compliance to ERAS in the subgroup of young patients. Consistent with the regression analysis, at each period after surgery, young high compliers were significantly more likely to benefit from adjuvant chemotherapy. For robustness, we also performed the analyses with interactions of ASA score and age. Those analyses showed that young ASA1-2 patients had a reduction in the delay of 10 days $(p=0.002)$ and ASA3 patients of 22 days $(p=0.021)$, indicating stronger effects in the higher ASA subgroup.

\section{Subgroup analyses_liver malignancies}

Liver colorectal metastases were analysed separately. A reduction of 14 days in the delay to adjuvant chemotherapy was found in young high compliers $(p=0.043)$ (Table 4).

\section{Subgroup analyses-pancreas malignancies}

Separate analyses concerning pancreas malignancies could not identify a significant effect of ERAS compliance on the time to receipt of adjuvant chemotherapy.

\section{Time to recurrence}

\section{Liver malignancies}

Young ASA3 patients with liver colorectal metastases and high compliance to ERAS had an improvement of diseasefree survival of 481 days $(p<0.001)$ (Table 5).

High compliance to ERAS in patients with liver colorectal metastases was not associated with a significant improvement of OS on multivariate analyses. Subgroup analysis of pancreas malignancies also did not show a significant impact of ERAS compliance on DFS and OS.

\section{Discussion}

The results of this study confirmed the positive effect of high compliance to ERAS regarding the post-operative outcomes in patients with hepatobiliary and pancreatic malignancies. In particular, an association between high ERAS compliance and a reduction in the time to receipt of adjuvant chemotherapy was identified in the subgroup of young patients $(<65$ years) whatever their comorbidities.

To our knowledge, no published study has assessed the relationship between ERAS compliance and the delay to adjuvant treatment for pancreatic and liver malignancies. It seems more likely that this delay would mainly be related to post-operative complications [19, 20, 24].

$\mathrm{Wu}$ et al., in a retrospective study including more than a thousand patients with pancreas ductal adenocarcinoma, found that complications after surgery were associated with a lower probability to have adjuvant oncological treatment and longer delay to adjuvant chemotherapy (median difference of 7 days). Moreover, they also found a correlation between post-operative complications and lower survival, but no clear relationship between delayed chemotherapy and decreased survival [19]. Petermann et al. had similar results, with lower survival related with post-operative complications after
Table 3 Time to chemotherapy — by age subgroup multivariate analysis (all patients)

\begin{tabular}{lllllll}
\hline Age (years) & Chg. Med. time & Std. err. & $z$ & $P>z$ & \multicolumn{2}{c}{$95 \%$ conf. interval } \\
\hline$<65$ & -12.089 & 3.673 & -3.290 & 0.001 & -19.287 & -4.891 \\
$\geq 65$ & 0.869 & 4.121 & 0.210 & 0.833 & -7.207 & 8.946 \\
\hline
\end{tabular}

Multivariate analysis of time to chemotherapy for all the study population, interacted with age

Chg. Med. Time change in median time, Std. Err standard error, 95\% Conf. Interval 95\% confidence interval 
No Adj. Chemo: Age<65

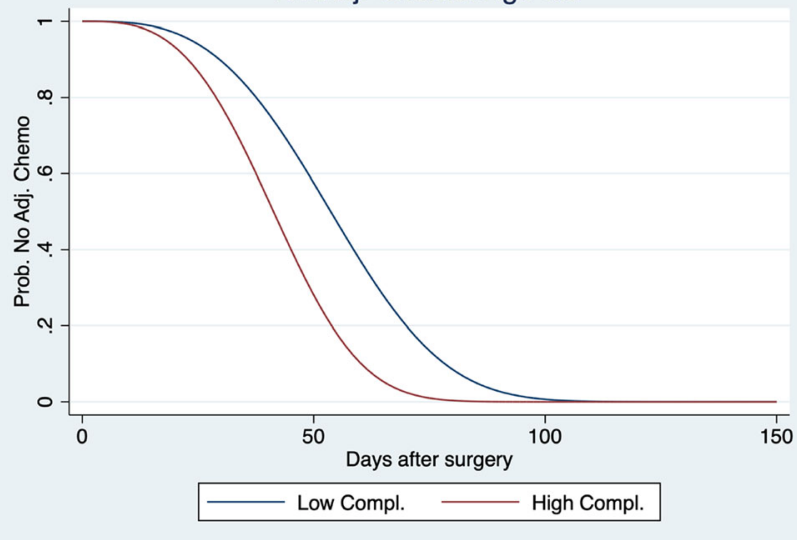

Fig. 3 Probability no adjuvant chemotherapy for young patients. Weibull distribution of the probability not to have an adjuvant chemotherapy at each moment after surgery in days, for high compliers (compliance $\geq$ $67 \%$ ) versus other compliances and for young patients (<65y.o.) with hepatobiliary and pancreas malignancies. Note that for this graph, $n$ Low Compl. $=40$ and $n$ High Comp. $=15, p=0.001$. Adj chemo adjuvant chemotherapy, Compl compliance, Prob probability

R1 resection of pancreatic head adenocarcinoma [25]. Kamphues et al. also found the same results in patients with pancreatic head tumours [26]. On the other hand, Murakami et al., in a retrospective study of 100 patients, concluded that post-operative complications were associated with delayed adjuvant chemotherapy for pancreatic carcinoma. They also showed evidence that early initiation of adjuvant treatment ( $<20$ days after surgery) was associated with a higher DFS and OS [24].

Valle et al., in a derived study of the prospective randomized trial ESPAC-3, with almost one thousand patients with pancreatic adenocarcinoma, found that delayed adjuvant chemotherapy up to 12 weeks for patients with completed cycles of treatment was not associated with lower overall survival [2]. In the study by Mirkin et al., comparing a delay to chemotherapy of more or less than 12 weeks, adjuvant chemotherapy was an independent prognostic factor in patients operated for pancreatic cancer, regardless of the time of initiation [3]. Similarly, in a retrospective study of almost nine hundred patients with pancreatic adenocarcinoma, adjuvant chemotherapy was found to be an independent prognostic factor, without any negative effect of a delayed treatment after more than 12 weeks post-operatively [20]. Finally, Nakagawa et al. also suggested that completion of adjuvant chemotherapy is a main determinant of long-term survival [27]. Therefore, results are conflicting and it is not clear, for hepatobiliary and pancreatic surgery, whether the delay to adjuvant chemotherapy is more important than the completion of all cycles, whatever the delay between surgery and the start of chemotherapy might be. However, it seems clear that it is more important to start an adjuvant treatment in a fit patient in order to complete all cycles of chemotherapy rather than starting too early in an unfit patient who would not tolerate the full cycles. The performance status of the patient with pancreatic adenocarcinoma is one of the main points in the decision to start adjuvant chemotherapy, which offers better outcomes [28].

In colorectal cancer, there are no studies analysing the effect of delayed chemotherapy on survival in patients with liver metastases. In a systematic review and meta-analysis, Biagi et al. concluded that delayed adjuvant chemotherapy in colorectal cancer, all stages confound, was associated with lower survival [4]. Kang et al. assessed the feasibility of initiating adjuvant chemotherapy before hospital discharge after surgery for stage II-IV colon cancer. They found no difference in cycle completion rates of chemotherapy for the two groups (in-hospital initiation vs outpatients). On the other hand, they found no significant difference in OS for patients with initiation of chemotherapy before hospital discharge compared with the other ones, but initiation of the treatment within 6 weeks was associated with better prognosis [5]. A systematic review and metaanalysis of outcomes in delayed adjuvant chemotherapy $(>6-$ 8 weeks) including gastric, colorectal and pancreatic cancers highlighted that a delayed chemotherapy was associated with a decreased survival for gastric and colorectal tumours, but not clearly for pancreatic malignancies [6].

Thus, it is recommended that adjuvant chemotherapy should be proposed for pancreatic cancer when indicated, even if delayed, and that a main point of the outcome improvement is the reduction of post-operative complications. Therefore, ERAS pathways implementation, by reducing post-operative complications, is certainly a key element to improve survival outcomes. Indeed, our study identified reduced delay to chemotherapy in young healthy and nonhealthy patients. This positive effect was not observed in elderly. This might be related to the initial functional status of elderly patients that preclude in some cases the proper implementation of all ERAS items. One can argue that younger and
Table 4 Time to chemotherapy — by age subgroup multivariate analysis (liver colorectal metastases)

\begin{tabular}{lllllll}
\hline Age (years) & Chg. Med. time & Std. err. & $z$ & $P>z$ & \multicolumn{2}{c}{$95 \%$ conf. interval } \\
\hline$<65$ & -13.492 & 6.677 & -2.020 & 0.043 & -26.578 & -0.405 \\
$\geq 65$ & 7.491 & 7.882 & 0.950 & 0.342 & -7.957 & 22.939 \\
\hline
\end{tabular}

Multivariate analysis of time to chemotherapy for patients with liver colorectal metastases, interacted with age Chg. Med. Time change in median time, Std. Err standard error, 95\% Conf. Interval 95\% confidence interval 
Table 5 Time to recurrence - by ASA and age subgroup multivariate analysis (liver colorectal metastases)

\begin{tabular}{llllllll}
\hline ASA & Age (years) & Chg. med. time & Std. err. & $z$ & $P>z$ & \multicolumn{2}{l}{$95 \%$ conf. interval } \\
\hline $1-2$ & $<65$ & -58.004 & 83.506 & -0.690 & 0.487 & -221.672 & 105.664 \\
$1-2$ & $\geq 65$ & 15.377 & 140.417 & 0.110 & 0.913 & -259.835 & 290.588 \\
3 & $<65$ & 480.889 & 130.812 & 3.680 & 0.000 & 224.502 & 737.277 \\
3 & $\geq 65$ & 831.072 & 520.342 & 1.600 & 0.110 & -188.781 & 1850.924 \\
\hline
\end{tabular}

Multivariate analysis of time to recurrence for patients with liver colorectal metastases, interacted with age and ASA score

ASA American Society of Anesthesiologists, Chg. Med. Time change in median time, Std. Err standard error, 95\% Conf. Interval $95 \%$ confidence interval fitter patients demonstrate a higher compliance with the entirety of recommended therapy including ERAS and multimodal oncologic therapy. Vice versa, frail or incompliant patients may have a lower compliance with both ERAS and adjuvant therapy. However, our data showed that compliance to ERAS protocol was comparable in patients $<65$ years old and $>65$ years old. Moreover, it was also comparable regarding the ASA score and the CCI score.

Despite similar rates of high compliers between young and old patients, we found a stronger effect in young patients, whatever the severity of their comorbidities. This could be related to a greater reduction in post-operative complications and increased opportunity to access multimodal oncologic therapies. This needs to be addressed and confirmed in future trials.

Finally, we found better compliance to ERAS items in preadmission and pre- and intra-operative than post-operative. Reasons for low compliance remain not clearly identified, but the global effect of ERAS implementation is well demonstrated, with reduced post-operative complications (principally medical ones) and length of stay. It is thought that many other unknown factors are implied in this relationship [29].

This study has several limitations inherited from its retrospective design and inclusion of patients with different types of cancer (small number of observations when analysed separately). Indeed, we analysed the marginal effect of compliance on a subgroup of young patients, interacted with ASA score to take into account pre-operative comorbidities. The effect of high compliance to ERAS remained significant to reduce the delay to adjuvant chemotherapy, when compared with low compliers. Thus, the results confirm that high compliance to ERAS protocol has a positive impact on complications and the delay to chemotherapy in a subgroup of young patients, and it will pave the way to further studies using strict ERAS protocol in liver and pancreas cancer. This will allow benchmarking and better comparisons between studies. with a reduction in the delay to adjuvant chemotherapy, particularly in young patients $<65$ years old. Moreover, young ASA3 patients with liver colorectal metastases and high compliance to ERAS had an increased DFS. Further studies are then needed to confirm these results.

Author's contributions Study conception and design: Pe. St-Amour, E. Melloul, D. Roulin. Acquisition of data: Pe. St-Amour, I. Labgaa, G-R. Jolliat, A. Eckert. Analysis and interpretation of data: Pe. St-Amour, Pa. St-Amour, E. Melloul. Drafting of manuscript: Pe. St-Amour, E. Melloul. Critical revision of manuscript: Pe. St-Amour, Pa. St-Amour, E. Melloul, N. Demartines

Funding Open access funding provided by University of Lausanne.

\section{Compliance with ethical standards}

Conflict of interest The authors declare that they have no conflict of interest.

Ethical approval All procedures performed in studies involving human participants were in accordance with the ethical standards of the institutional and/or national research committee and with the 1964 Helsinki declaration and its later amendments or comparable ethical standards.

Informed consent Informed consent obtained from all individual participants included in the study.

Open Access This article is licensed under a Creative Commons Attribution 4.0 International License, which permits use, sharing, adaptation, distribution and reproduction in any medium or format, as long as you give appropriate credit to the original author(s) and the source, provide a link to the Creative Commons licence, and indicate if changes were made. The images or other third party material in this article are included in the article's Creative Commons licence, unless indicated otherwise in a credit line to the material. If material is not included in the article's Creative Commons licence and your intended use is not permitted by statutory regulation or exceeds the permitted use, you will need to obtain permission directly from the copyright holder. To view a copy of this licence, visit http://creativecommons.org/licenses/by/4.0/.

\section{Conclusion}

To conclude, high compliance to ERAS in oncologic hepatobiliary and pancreatic surgery tends to be associated

\section{References}

1. Siegel RL, Miller KD, Jemal A (2019) Cancer statistics, 2019. CA Cancer J Clin 69(1):7-34 
2. Valle JW, Palmer D, Jackson R, Cox T, Neoptolemos JP, Ghaneh P, Rawcliffe CL, Bassi C, Stocken DD, Cunningham D, O'Reilly D, Goldstein D, Robinson BA, Karapetis C, Scarfe A, Lacaine F, Sand J, Izbicki JR, Mayerle J, Dervenis C, Oláh A, Butturini G, Lind PA, Middleton MR, Anthoney A, Sumpter K, Carter R, Büchler MW (2014) Optimal duration and timing of adjuvant chemotherapy after definitive surgery for ductal adenocarcinoma of the pancreas: ongoing lessons from the ESPAC-3 study. J Clin Oncol 32(6):504512

3. Mirkin KA, Greenleaf EK, Hollenbeak CS, Wong J (2016) Time to the initiation of adjuvant chemotherapy does not impact survival in patients with resected pancreatic cancer. Cancer. 122(19):29792987

4. Biagi JJ, Raphael MJ, Mackillop WJ, Kong W, King WD, Booth CM (2011) Association between time to initiation of adjuvant chemotherapy and survival in colorectal cancer: a systematic review and meta-analysis. JAMA. 305(22):2335-2342

5. Kang J, Chong SW, Park EJ, Baik SH, Lee KY (2019) Safety and feasibility of in-hospital early chemotherapy initiation after surgery in patients with stage II-IV colon cancer. Medicine (Baltimore) 98(18):e15371

6. Petrelli F, Zaniboni A, Ghidini A, Ghidini M, Turati L, Pizzo C, et al. (2019) Timing of adjuvant chemotherapy and survival in colorectal, gastric, and pancreatic cancer. A systematic review and meta-analysis. Cancers (Basel);11(4)

7. Ji HB, Zhu WT, Wei Q, Wang XX, Wang HB, Chen QP (2018) Impact of enhanced recovery after surgery programs on pancreatic surgery: a meta-analysis. World J Gastroenterol 24(15):1666-1678

8. Yang R, Tao W, Chen YY, Zhang BH, Tang JM, Zhong S et al (2016) Enhanced recovery after surgery programs versus traditional perioperative care in laparoscopic hepatectomy: a meta-analysis. Int J Surg 36(Pt A):274-282

9. Zhao Y, Qin H, Wu Y, Xiang B (2017) Enhanced recovery after surgery program reduces length of hospital stay and complications in liver resection: a PRISMA-compliant systematic review and meta-analysis of randomized controlled trials. Medicine (Baltimore) 96(31):e7628

10. Mahendran R, Tewari M, Dixit VK, Shukla HS (2019) Enhanced recovery after surgery protocol enhances early postoperative recovery after pancreaticoduodenectomy. Hepatobiliary Pancreat Dis Int 18(2):188-193

11. Li L, Chen J, Liu Z, Li Q, Shi Y (2017) Enhanced recovery program versus traditional care after hepatectomy: a meta-analysis. Medicine (Baltimore) 96(38):e8052

12. Lassen K, Coolsen MM, Slim K, Carli F, de Aguilar-Nascimento JE, Schafer M et al (2012) Guidelines for perioperative care for pancreaticoduodenectomy: Enhanced Recovery After Surgery (ERAS(R)) Society recommendations. Clin Nutr 31(6):817-830

13. Melloul E, Hubner M, Scott M, Snowden C, Prentis J, Dejong CH et al (2016) Guidelines for perioperative care for liver surgery: Enhanced Recovery After Surgery (ERAS) Society Recommendations. World J Surg 40(10):2425-2440

14. Melloul E, Lassen K, Roulin D, Grass F, Perinel J, Adham M, et al. (2019) Guidelines for perioperative care for pancreatoduodenectomy: Enhanced Recovery After Surgery (ERAS) Recommendations. World J Surg. 2020

15. Kitahata Y, Hirono S, Kawai M, Okada KI, Miyazawa M, Shimizu A, Kobayashi R, Ueno M, Hayami S, Shimokawa T, Kouda K, Tajima F, Yamaue H (2018) Intensive perioperative rehabilitation improves surgical outcomes after pancreaticoduodenectomy. Langenbeck's Arch Surg 403(6):711-718
16. Mise Y, Vauthey JN, Zimmitti G, Parker NH, Conrad C, Aloia TA, Lee JE, Fleming JB, Katz MHG (2015) Ninety-day postoperative mortality is a legitimate measure of hepatopancreatobiliary surgical quality. Ann Surg 262(6):1071-1078

17. Goussous N, Hosseini M, Sill AM, Cunningham SC (2017) Minimally invasive and open gallbladder cancer resections: 30 vs 90-day mortality. Hepatobiliary Pancreat Dis Int 16(4):405-411

18. Swanson RS, Pezzi CM, Mallin K, Loomis AM, Winchester DP (2014) The 90-day mortality after pancreatectomy for cancer is double the 30-day mortality: more than 20,000 resections from the national cancer data base. Ann Surg Oncol 21(13):4059-4067

19. Wu W, He J, Cameron JL, Makary M, Soares K, Ahuja N, Rezaee N, Herman J, Zheng L, Laheru D, Choti MA, Hruban RH, Pawlik TM, Wolfgang CL, Weiss MJ (2014) The impact of postoperative complications on the administration of adjuvant therapy following pancreaticoduodenectomy for adenocarcinoma. Ann Surg Oncol 21(9):2873-2881

20. Xia BT, Ahmad SA, Al Humaidi AH, Hanseman DJ, Ethun CG, Maithel SK et al (2017) Time to initiation of adjuvant chemotherapy in pancreas cancer: a multi-institutional experience. Ann Surg Oncol 24(9):2770-2776

21. Sugiura T, Okamura Y, Ito T, Yamamoto Y, Ashida R, Uesaka K (2017) Impact of patient age on the postoperative survival in pancreatic head cancer. Ann Surg Oncol 24(11):3220-3228

22. Li X, Liu Z, Ye Z, Gou S, Wang C (2018) Impact of age on survival of patients with pancreatic cancer after surgery: Analysis of SEER data. Pancreatology. 18(1):133-138

23. Bos AC, van Erning FN, van Gestel YR, Creemers GJ, Punt CJ, van Oijen MG et al (2015) Timing of adjuvant chemotherapy and its relation to survival among patients with stage III colon cancer. Eur J Cancer 51(17):2553-2561

24. Murakami Y, Uemura K, Sudo T, Hashimoto Y, Kondo N, Nakagawa N, Sasaki H, Sueda T (2013) Early initiation of adjuvant chemotherapy improves survival of patients with pancreatic carcinoma after surgical resection. Cancer Chemother Pharmacol 71(2): 419-429

25. Petermann D, Demartines N, Schafer M (2013) Severe postoperative complications adversely affect long-term survival after R1 resection for pancreatic head adenocarcinoma. World J Surg 37(8): 1901-1908

26. Kamphues C, Bova R, Schricke D, Hippler-Benscheidt M, Klauschen F, Stenzinger A, Seehofer D, Glanemann M, Neuhaus P, Bahra M (2012) Postoperative complications deteriorate longterm outcome in pancreatic cancer patients. Ann Surg Oncol 19(3): $856-863$

27. Nakagawa K, Akahori T, Nishiwada S, Nagai M, Nakamura K, Tanaka T, Tamamoto T, Ohbayashi C, Hasegawa M, Kichikawa K, Ikeda N, Sho M (2018) Prognostic factors for actual long-term survival in the era of multidisciplinary treatment for pancreatic ductal adenocarcinoma. Langenbeck's Arch Surg 403(6):693-700

28. Klaiber U, Leonhardt CS, Strobel O, Tjaden C, Hackert T, Neoptolemos JP (2018) Neoadjuvant and adjuvant chemotherapy in pancreatic cancer. Langenbeck's Arch Surg 403(8):917-932

29. Roulin D, Melloul E, Wellg BE, Izbicki J, Vrochides D, Adham M, Hübner M, Demartines N (2020) Feasibility of an enhanced recovery protocol for elective pancreatoduodenectomy: a multicenter international cohort study. World J Surg 44:2761-2769

Publisher's note Springer Nature remains neutral with regard to jurisdictional claims in published maps and institutional affiliations. 